\title{
In Situ High Temperature Atomic Resolution Transmission Electron Microscopy of 2D Nanomaterials
}

\author{
Ashley L. Gibb ${ }^{1,2,3}$, Nasim Alem ${ }^{4,5}$, Jian-Hao Chen ${ }^{2,6}$, Jim Ciston $^{4}$, and Alex Zettl ${ }^{2,3}$ \\ ${ }^{1}$ Department of Chemistry, University of California Berkeley, Berkeley, CA USA \\ ${ }^{2}$ Department of Physics, University of California Berkeley, Berkeley, CA USA \\ ${ }^{3}$ Materials Science Division, Lawrence Berkeley National Lab, Berkeley CA USA \\ ${ }^{4}$ National Center for Electron Microscopy, Berkeley, CA USA \\ ${ }^{5}$ Department of Materials Science, Pennsylvania State University, State College, PA USA \\ ${ }^{6}$ Department of Physics, Peking University, Beijing, China
}

Two-dimensional hexagonal nanomaterials have gained substantial research attention for their interesting properties. In the case of graphene, a single atom thick layer of carbon, focus has been on its high electron mobility and mechanical strength for applications in touch-screens, sensors, batteries, and other devices[1]. Hexagonal boron nitride, another single atom thick $\mathrm{sp}^{2}$ hexagonal nanomaterial, is structurally isoelectronic with graphene. It has a band gap greater than $5 \mathrm{eV}$, exhibits high thermal conductivity, high mechanical strength and chemical stability[2]. Due to being atomically flat and electrically insulating, h-BN has proven to be an excellent substrate for graphene devices. Hexagonal boron nitride also exhibits resistance to oxidation and stability up to approximately $1500{ }^{\circ} \mathrm{C}$ in air, making it an excellent material for high temperature coatings[3]. Additionally, it has many promising applications in the aerospace, textiles, medical, and electronics industries. Scalable growth of mono- to few-layer hexagonal boron nitride is achievable using chemical vapor deposition from borazine or ammonia borane [4,5]. However, for many applications, nanoscale and atomic scale defects can impact the performance of the material. In this abstract, we report on the structure of defects in hexagonal boron nitride at elevated temperature using aberration corrected transmission electron microscopy (TEM).

Hexagonal boron nitride was grown using low pressure chemical vapor deposition conditions reported previously using nickel foil $(0.025 \mathrm{~mm}$ thick, $99.99 \%$, Alfa Aesar) as the catalytic substrate for growth [6]. Growth occurred at $1173 \mathrm{~K}$ with a flow of hydrogen gas using chilled borazine (Gelest Inc.) as a precursor. The h-BN was transferred to an Aduro heating stage (Protochips Inc.) for TEM analysis. Transmission electron microscopy was performed using the TEAM 0.5 microscope operating at $80 \mathrm{kV}$, yielding a spatial resolution of less than $1 \AA$

A variety of defects were observed in h-BN, most commonly triangular vacancy defects. These vacancies are typically formed when a boron atom is ejected from the lattice due to electron beam induced knock on damage. Theoretical calculations have predicted that vacancy defects in boron nitride are completely immobile until $840 \mathrm{~K}$ [7]. However, previous studies of vacancy migration at room temperature have found that the energy from the electron beam during TEM imaging is sufficient to overcome this energy barrier [8]. In graphene, previous studies have found that under electron beam irradiation mobile carbon atoms and vacancies can allow holes and defects to heal, or regrow into the graphene lattice [9].

In this experiment, we heated the suspended h-BN sample to $973 \mathrm{~K}$ and observed vacancy formation, migration, coalescence, and healing. All vacancies formed at this temperature are triangular in shape and oriented in a single direction along the lattice. However, compared to previous studies at room 
temperature, triangular defects at $973 \mathrm{~K}$ coalesce more quickly and migrate more readily [7]. This migration is due to atoms along the edges of defects which are destabilized and become mobile. These edge atoms are either eliminated by further electron beam damage or can move to different defects. Over time, the vacancy defects coalesce. Single isolated vacancies appear to consistently grow until they are within 1-2 nm of a larger defect, at which point they "heal" and become smaller in size until finally coalescing with the larger defect. In Figure 1, several triangular vacancies and many mono-vacancies can be observed which grow in size until within $1 \mathrm{~nm}$ of the approaching edge, at which point they begin to coalesce and in some cases partially heal.
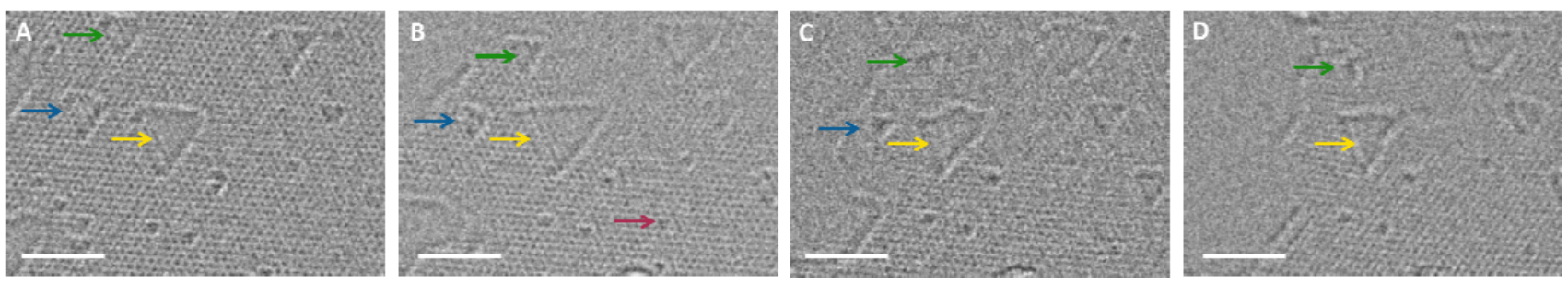

Figure 1. Transmission electron microscope image of hexagonal boron nitride at $973 \mathrm{~K}$. Scale bars $2 \mathrm{~nm}$. A. At $\mathrm{t}=0$ seconds; B. At $\mathrm{t}=6$ seconds. Central triangle defect (marked by yellow arrow) has grown in size, while left most defect (blue arrow) has become smaller; C. At $t=9$ seconds. As the edge approaches, the defects with blue, yellow and green arrows have reduced in size, while the right most defects have grown. The mono-vacancy from B (red arrow) has healed or migrated out of field of view.; D. At $t=12$ seconds. The blue defect has merged with the edge, and the yellow and green defects have decreased in size.

In conclusion, we observed defect formation, migration, and healing in h-BN at elevated temperature. The rate of vacancy migration and size changes appears to depend on their proximity to larger holes. Further studies of vacancy dynamics in h-BN at various temperatures are needed to further understand the rates and mechanisms of this formation.

\section{References:}

[1] Geim, A. K.; Novoselov, K. S. Nat. Mater. 6, 183. (2007).

[2] Golberg, D. et al. ACS Nano 4, 2979 (2010).

[3] Liu, Z. et al. Nat. Comm. 4, 2541 (2013).

[4] Gibb, A. et al. Phys. Status Solidi B. 250, 2727 (2013).

[5] K. K. Kim, et al, ACS Nano 6, 8583 (2012).

[6] Gibb, A. et al. JACS 135, 6758 (2013).

[7] Zobelli, A. et al. Phys. Rev. B. 75, 245402 (2007).

[8] Alem, N. et al. Phys. Status Solidi RRL 5, 295 (2011).

[9] Zan, R. et al. Nano Lett. 12, 3936 (2012).

[10] This work was supported in part by the Director, Office of Energy Research, Office of Basic Energy Sciences, Materials Sciences and Engineering Division, of the U.S. Department of Energy under Contract \#DE-AC02-05CH11231, the Office of Naval Research under MURI award N00014-09-1-1066; and the Air Force Office of Scientific Research under grant \#FA9950-10-1-0451. Transmission electron microscopy work was performed at the National Center for Electron Microscopy; AG acknowledges support from an NSF graduate research fellowship. 